\title{
An X-ray halo in the "hot-spot” galaxy NGC 2903
}

\author{
D. Tschöke ${ }^{1}$, G. Hensler ${ }^{1}$, and N. Junkes ${ }^{2}$ \\ 1 Institut für Theoretische Physik und Astrophysik, Universität Kiel, 24098 Kiel, Germany \\ 2 Max-Planck-Institut für Radioastronomie, Auf dem Hügel 69, 53121 Bonn, Germany
}

Received 2 February 2001/ Accepted 16 June 2003

\begin{abstract}
In this paper we present ROSAT PSPC and HRI observations of the "hot-spot" galaxy NGC 2903. This isolated system strikingly reveals a soft extended X-ray feature reaching in north-west direction up to a projected distance of $5.2 \mathrm{kpc}$ from the center into the halo. The residual X-ray emission in the disk reveals the same extension as the $\mathrm{H} \alpha$ disk. No eastern counterpart of the western X-ray halo emission has been detected. The luminosity of the extraplanar X-ray gas is several $10^{38} \mathrm{erg} \mathrm{s}^{-1}$, comparable to X-ray halos in other starburst galaxies. It has a plasma temperature of about $0.2 \mathrm{keV}$. The estimated star formation rate derived from X-rays and $\mathrm{H} \alpha$ results in 1-2 $M_{\odot} \mathrm{yr}^{-1}$. Since galactic superwinds, giant kpc-scale galactic outflows, seem to be a common phenomenon observed in a number of edge-on galaxies, especially in the X-ray regime, and are produced by excess star-formation activity, the existence of hot halo gas as found in NGC 2903 can be attributed to events such as central starbursts. That such a starburst has taken place in NGC 2903 must be proven. The detection of hot gas above galaxy disks also with intermediate inclination, however, encounters the difficulty of discriminating between that contribution from disk and active nuclear region.
\end{abstract}

Key words. galaxies: active - galaxies: starburst - galaxies: ISM - galaxies: individual: NGC 2903 - X-rays: galaxies

\section{Introduction}

Starbursts are important mechanisms for the chemical and dynamical evolution of galaxies and the chemical enrichment of the intergalactic medium (IGM). Mass exchange between different galactic components (stars - interstellar medium (ISM) clouds) and also between galactic disks, halo and the environmental IGM strongly influence the nature of these systems and occur on rather short timescales. Typical durations of starbursts are up to a few $10^{7} \mathrm{yr}$. Observations in different spectral ranges can trace different evolutionary phases of starbursts and components involved. Supernova remnants (SNRs) are detectable by non-thermal radio continuum, by both X-ray line and continuum emission as well as by infrared lines; strong infrared continumm sources reflect huge amounts of dust heated by young massive stars; giant extragalactic HII regions in the optical and strong UV fluxes of OB associations are other tracers of the actual SF (Shields 1990).

Our interest is concerning the hot components observable in the X-ray regime. Stellar winds from young massive O- and B-type stars heat up the ambient ISM by shocks (Leitherer 1994; Freyer \& Hensler 2000). These stars end in supernova explosions of type II (SNe II), which expand into the ISM. Due to the strong spatial concentration of OB associations and because of the short evolutionary timescale of

Send offprint requests to: G. Hensler, e-mail: hensler@astrophysik.uni-kiel.de massive stars, the subsequent SNe II accumulate to build socalled superbubbles, hot cavities in the ISM filled with several million Kelvin hot plasma. These expand due to their overpressure and preferentially perpendicularly to a galactic disk, because of the steeper density gradient. If enough energy from the starburst is converted into kinetic energy of the propagating hot gas, it breaks out of the disk into the galactic halo (Norman \& Ikeuchi 1989; Heckman et al. 1990; Suchkov et al. 1994; Strickland \& Stevens 2000). These hot gaseous outflows or superwinds have been detected in the soft X-rays in several edge-on disk galaxies (see e.g. Dahlem et al. 1998, hereafter DWH98) and must be a common phenomenon from the theoretical point of view.

We have investigated a small sample of spiral galaxies with nuclear starburst activity (NGC 1808: Junkes et al. 1995; NGC 4410: Tschöke et al. 1999; NGC 4303: Tschöke et al. 2000; NGC 4569: Bomans et al. 2002; Tschöke et al. 2001) in order to obtain insights into the processes that enhance SF and to probe their evolution. These hot gaseous outflows are rather difficult to be directly observed. The best candidates are edgeon spiral galaxies with significant SF where any X-ray component outside the disk can be well distinguished, and where a sufficient amount of hot gas can be detected. Most prominent examples are the two closest starburst galaxies, M 82 and NGC 253 (Weaver et al. 2000; Pietsch et al. 2000). In contrast, for the X-ray emission of galaxies observed at low or intermediate inclination angles the location of the diffuse component, 
Table 1. NGC 2903: Some basic parameters.

\begin{tabular}{lcc}
\hline \hline & NGC 2903 & Reference $^{a}$ \\
\hline Alternate name & IRAS 09293+2143 & \\
Type & SAB $(\mathrm{rs}) \mathrm{bc}$ & 1 \\
RA $(2000)$ & $09^{\mathrm{h}} 32^{\mathrm{m}} 09^{s} .9$ & 2 \\
Dec $(2000)$ & $+21^{\circ} 30^{\prime} 07^{\prime \prime}$ & 2 \\
Distance/Mpc & 8.9 & 3 \\
Radial velocity $/ \mathrm{km} \mathrm{s}^{-1}$ & 556 & 3 \\
$D_{25}$ diameter & $11^{\prime} .6$ & 1 \\
Axial ratio & 0.49 & 1 \\
Inclination & $66^{\circ}$ & 1 \\
$\log \left(L_{\mathrm{B}} / L_{\odot}\right)$ & 10.13 & 1 \\
$\log \left(M_{\mathrm{HI}} / M_{\odot}\right)$ & 9.25 & 1 \\
$\log \left(N_{\mathrm{HI}} / \mathrm{cm}^{-2}\right)($ Galactic $)$ & 20.45 & 4 \\
$\log \left(N_{\mathrm{HI}} / \mathrm{cm}^{-2}\right)($ intrinsic $)$ & 20.92 & 5 \\
\hline
\end{tabular}

${ }^{a}$ References:

1) Tully (1988).

2) Falco et al. (1999).

3) Drozdovsky et al. (2000).

4) Dickey \& Lockman (1990).

5) Wevers et al. (1986).

whether within or above the disk, is mostly indistinguishable. Moreover, soft (typically $0.2 \mathrm{keV}$ ) X-rays from such gaseous outflows to behind the galactic disk are usually extinguished.

One candidate of a galaxy at intermediate inclination with an observed X-ray halo is NGC 4569 (Tschöke et al. 2001). Another one is NGC 2903, a nearby barred galaxy where most of the investigations so far have been concerned with its nucleus containing a central starburst. NGC 2903 has been classified as "hot-spot" galaxy (Sérsic 1973) because of the complex optical structure of the nucleus (Oka et al. 1974; Laques et al. 1980; Simons et al. 1988; Planesas et al. 1997) that shows six separate emission peaks over an area of about $20^{\prime \prime}$ in diameter. Radio maps of the nuclear region do not exactly correspond to the optical hot spots. As an explanation, Wynn-Williams \& Becklin (1985) suggested the possibility of patchy extinction. Recent near-infrared observations of the galactic core indeed reveal a ring-like morphology with a diameter of 625 pc (Alonso-Herrero et al. 2001), coinciding with the radio rather than with the $\mathrm{H} \alpha$ structure. The nucleus contains a strong non-thermal radio source (van der Kruit 1973), reflecting SNRs in the nuclear starburst, and a moderately high FIR luminosity of $\leq 7 \times 10^{9} L_{\odot}$ (Telesco \& Harper 1980). H $\alpha$ images of the whole galaxy clearly reveal the bar and spiral structure with a dominant central source (Jackson et al. 1991). A few basic properties of NGC 2903 are listed in Table 1.

In this paper we present spectral and imaging data of NGC 2903 obtained by the X-ray satellite ROSAT in the 0.1$2.4 \mathrm{keV}$ band. We discuss the contributions from different components of NGC 2903 (nucleus, disk, and halo) in order to achieve a more complete picture of SF processes in this nuclear starburst galaxy. Section 2 presents the observation protocol and reduction techniques of the used ROSAT data, Sect. 3 the results from imaging and spectral analysis. In Sect. 4 we discuss the derived X-ray properties of NGC 2903, compare the derived SF rate with results from other wavelengths, and confirm the existence of an extraplanar X-ray gas component above the galactic disk. The results are summarized in Sect. 5. Throughout the paper we adopt the recent photometric distance estimation of 8.9 Mpc for NGC 2903 (Drozdovsky et al. 2000). Hence 1 " corresponds to $43 \mathrm{pc}$.

\section{Observations and data reduction}

The presented data were taken by the High Resolution Imager (HRI) and the Position Sensitive Proportional Counter (PSPC) onboard the X-ray satellite ROSAT. For details concerning ROSAT and its detectors see the ROSAT User's Handbook (Briel et al. 1996). Some basic information on the observations are given in Table 2. Source detection (Tables 3 and 4) was performed with the EXSAS software package (Zimmermann et al. 1997). Images and spectra were created by using the Imaging Data Language (IDL) software package. To fit the spectral distribution of the X-ray source we used XSPEC (Arnaud 1996).

\section{1. $H R I$}

Two separate exposures were taken with the HRI detector (see Table 2). The data were combined, creating one exposure of $21.9 \mathrm{ksec}$, corrected for vignetting, but not for background contamination. The resulting image (Fig. 1a) was convolved with a Gaussian of 5" FWHM. To determine the background level, three source-free circular fields around NGC 2903 were chosen, with radii of $93^{\prime \prime}, 79^{\prime \prime}$, and $60^{\prime \prime}$, respectively.

The source detection in the HRI field of view (FOV) was carried out with a maximum likelihood method and a lower detection limit of $5 \sigma$ and is listed in Table 3.

\section{2. $P S P C$}

The PSPC exposure was centered on the same coordinates as the HRI observations. The created image (Fig. 1b) was corrected for vignetting and convolved with a Gaussian of $25^{\prime \prime}$. The background flux was determined from three circular source-free areas in the field with radii of 95", 71", and 59". The source detection in the FOV was carried out the same way as in the case of the HRI observations. Results are given in Table 4.

\section{Results}

\subsection{X-ray imaging}

The X-ray emission in the ROSAT PSPC image of NGC 2903 (Fig. 2) is clearly extended. It covers the whole inner region with the galactic bar and the inner spiral structure up to a radius of $5 \mathrm{kpc}$, showing a strong emission peak near the optical center and significant contributions from the northern and southern disk component. The northern nose-like elongation of the disk component particularly visible at intermediate energy contours (Fig. 2c) indicates a second X-ray peak coincident with a luminous HII region at about $2^{\prime}$ northeast from the center, also 
Table 2. ROSAT observations of NGC 2903.

\begin{tabular}{|c|c|c|c|c|c|c|c|c|}
\hline & \multicolumn{2}{|c|}{ Central coordinates } & \multirow[t]{2}{*}{ Date } & \multirow[t]{2}{*}{ ROR no. } & \multirow{2}{*}{$\begin{array}{l}\text { Exposure } \\
\text { time }[\mathrm{s}]\end{array}$} & \multirow{2}{*}{$\begin{array}{l}\text { Count rate } \\
{\left[\operatorname{cts~s}^{-1}\right]}\end{array}$} & \multirow{2}{*}{$\begin{array}{l}\text { Background } \\
{\left[\operatorname{cts~s}^{-1} \operatorname{arcsec}^{-2}\right]}\end{array}$} & \multirow[t]{2}{*}{$\overline{P I}$} \\
\hline & $\mathrm{RA}(2000)$ & $\operatorname{Dec}(2000)$ & & & & & & \\
\hline \multirow[t]{2}{*}{ HRI } & $9^{\mathrm{h}} 32^{\mathrm{m}} 9^{\mathrm{s}} .6$ & $+21^{\circ} 30^{\prime} 00^{\prime \prime}$ & April 27-29, 1994 & $600602 \mathrm{~h}$ & 13619 & $0.020^{b}$ & $3.6 \times 10^{-7 b}$ & N. Junkes \\
\hline & $9^{\mathrm{h}} 32^{\mathrm{m}} 9^{\mathrm{s}} .6$ & $+21^{\circ} 30^{\prime} 00^{\prime \prime}$ & Nov. 7, 1994 & dto. & 8284 & & & N. Junkes \\
\hline PSPC & $9^{\mathrm{h}} 32^{\mathrm{m}} 9^{\mathrm{s}} .6$ & $+21^{\circ} 30^{\prime} 00^{\prime \prime}$ & May 6-7, 1991 & $600093 p$ & 9184 & 0.091 & $3.2 \times 10^{-7}$ & H. Zinnecker \\
\hline
\end{tabular}

${ }^{a}$ Background subtracted.

${ }^{b}$ Combined HRI observations.

Table 3. Source detections in the ROSAT HRI observation of NGC 2903 with a maximum likelihood threshold of $14(5 \sigma)$.

\begin{tabular}{lllllll}
\hline \hline No. & RA (2000) & Dec (2000) & $\begin{array}{l}\text { Existence } \\
\text { likelihood }\end{array}$ & $\begin{array}{l}\text { Count rate (HRI) } \\
{\left[10^{-3} \mathrm{cts} \mathrm{s}^{-1}\right]}\end{array}$ & $\begin{array}{l}\text { No. } \\
\text { (PSPC) }\end{array}$ & Identification \\
\hline 1 & $9^{\mathrm{h}} 32^{\mathrm{m}} 05^{\mathrm{s}} .4$ & $+21^{\circ} 32^{\prime} 36^{\prime \prime}$ & 23 & $0.8 \pm 0.2$ & 9 & HII region in NGC 2903 \\
2 & $9^{\mathrm{h}} 33^{\mathrm{m}} 22^{\mathrm{s}} .2$ & $+21^{\circ} 32^{\prime} 07^{\prime \prime}$ & 18 & $3.3 \pm 0.7$ & 10 & 2E 0930.5+2145 \\
3 & $9^{\mathrm{h}} 32^{\mathrm{m}} 10^{\mathrm{s}} .1$ & $+21^{\circ} 30^{\prime} 07^{\prime \prime}$ & 180 & $7.8 \pm 0.6$ & 11 & NGC 2903 \\
4 & $9^{\mathrm{h}} 31^{\mathrm{m}} 48^{\mathrm{s}} .7$ & $+21^{\circ} 24^{\prime} 43^{\prime \prime}$ & 20 & $1.0 \pm 0.3$ & 14 & \\
\hline
\end{tabular}

${ }^{a}$ Background subtracted.

Table 4. Source detections in the ROSAT PSPC observation of NGC 2903 with a maximum likelihood threshold of $14(5 \sigma)$.

\begin{tabular}{|c|c|c|c|c|c|c|}
\hline No. & RA (2000) & $\overline{~ D e c ~(2000) ~}$ & $\begin{array}{l}\text { Existence } \\
\text { likelihood }\end{array}$ & $\begin{array}{l}\text { Count rate (PSPC) } \\
{\left[10^{-3} \mathrm{cts} \mathrm{s}^{-1}\right]}\end{array}$ & $\begin{array}{l}\text { No. } \\
\text { (HRI) }\end{array}$ & Identification \\
\hline 1 & $9^{\mathrm{h}} 32^{\mathrm{m}} 13^{\mathrm{s}} \cdot 2$ & $+21^{\circ} 51^{\prime} 35^{\prime \prime}$ & 31 & $7.6 \pm 1.4$ & & HD 82394/BD +22 2102B \\
\hline 2 & $9^{\text {h }} 32^{\mathrm{m}} 12^{\mathrm{s}} .9$ & $+21^{\circ} 48^{\prime} 12^{\prime \prime}$ & 20 & $4.4 \pm 1.0$ & & \\
\hline 3 & $9^{\mathrm{h}} 33^{\mathrm{m}} 14^{\S} .3$ & $+21^{\circ} 46^{\prime} 00^{\prime \prime}$ & 31 & $9.9 \pm 1.6$ & & \\
\hline 4 & $9^{\mathrm{h}} 31^{\mathrm{m}} 55^{\mathrm{s}} .2$ & $+21^{\circ} 43^{\prime} 58^{\prime \prime}$ & 19 & $4.4 \pm 1.0$ & & QSO 0929+2157 \\
\hline 5 & $9^{\mathrm{h}} 32^{\mathrm{m}} 33^{\mathrm{s}} .3$ & $+21^{\circ} 41^{\prime} 54^{\prime \prime}$ & 36 & $5.1 \pm 1.0$ & & \\
\hline 6 & $9^{\text {h }} 30^{\mathrm{m}} 56.5$ & $+21^{\circ} 34^{\prime} 34^{\prime \prime}$ & 37 & $6.7 \pm 1.2$ & & \\
\hline 7 & $9^{\text {h }} 32^{\mathrm{m}} 56^{\mathrm{s}} .3$ & $+21^{\circ} 33^{\prime} 57^{\prime \prime}$ & 23 & $3.5 \pm 0.8$ & & \\
\hline 8 & $9^{\mathrm{h}} 31^{\mathrm{m}} 25^{\mathrm{s}} \cdot 6$ & $+21^{\circ} 33^{\prime} 46^{\prime \prime}$ & 46 & $5.1 \pm 0.9$ & & \\
\hline 9 & $9^{\mathrm{h}} 32^{\mathrm{m}} 05^{\mathrm{s}} .5$ & $+21^{\circ} 32^{\prime} 33^{\prime \prime}$ & 33 & $3.6 \pm 0.8$ & 1 & HII region in NGC 2903 \\
\hline 10 & $9^{\mathrm{h}} 33^{\mathrm{m}} 21^{\mathrm{s}} .9$ & $+21^{\circ} 32^{\prime} 12^{\prime \prime}$ & 214 & $21.6 \pm 1.8$ & 2 & $2 \mathrm{E} 0930.5+2145$ \\
\hline 11 & $9^{\mathrm{h}} 32^{\mathrm{m}} 09.9$ & $+21^{\circ} 30^{\prime} 14^{\prime \prime}$ & 1157 & $73.8 \pm 3.0$ & 3 & NGC 2903 \\
\hline 12 & $9^{\mathrm{h}} 31^{\mathrm{m}} 20.7$ & $+21^{\circ} 29^{\prime} 44^{\prime \prime}$ & 19 & $3.0 \pm 0.8$ & & \\
\hline 13 & $9^{\text {h }} 30^{\mathrm{m}} 59^{\mathrm{s}} .9$ & $+21^{\circ} 29^{\prime} 09^{\prime \prime}$ & 18 & $3.7 \pm 0.9$ & & \\
\hline 14 & $9^{\mathrm{h}} 31^{\mathrm{m}} 48.7$ & $+21^{\circ} 24^{\prime} 48^{\prime \prime}$ & 19 & $2.6 \pm 0.7$ & 4 & \\
\hline 15 & $9^{\mathrm{h}} 31^{\mathrm{m}} 44^{\S} .4$ & $+21^{\circ} 17^{\prime} 04^{\prime \prime}$ & 33 & $5.0 \pm 1.0$ & & \\
\hline 16 & $9^{\mathrm{h}} 32^{\mathrm{m}} 06.3$ & $+21^{\circ} 15^{\prime} 03^{\prime \prime}$ & 17 & $4.7 \pm 1.1$ & & QSO $0929+2128$ \\
\hline 17 & $9^{\mathrm{h}} 33^{\mathrm{m}} 47^{\mathrm{s}} .9$ & $+21^{\circ} 14^{\prime} 33^{\prime \prime}$ & 376 & $55.2 \pm 3.3$ & & QSO $0930+2128$ \\
\hline 18 & $9^{\mathrm{h}} 30^{\mathrm{m}} 45^{\mathrm{s}} .5$ & $+21^{\circ} 02^{\prime} 17^{\prime \prime}$ & 50 & $18.7 \pm 2.5$ & & HD 82157 \\
\hline
\end{tabular}

${ }^{a}$ Background subtracted.

perceptible in the $\mathrm{H} \alpha$ image. More striking is the coincidence of the contour elongation with a prominent HII region $1^{\prime}$ north of the center that is also visible in the HRI (Fig. 3).

Another point source about 2!7 northwest from the center (Table 4, source No. 9) spatially coincides with an HII region in the norther spiral arm, supported by the coincidence in the HRI image (Fig. 3; see also Table 3, source No. 1). Furthermore, the spectral distribution is comparable with the one of the main disk emission (component No. 5 in Table 5; Fig. 2).

In Fig. 2 we directly compare the X-ray emission of NGC 2903 in four energy bands, the total spectral range of the PSPC $(0.1-2.4 \mathrm{keV})$, the soft range $(0.1-0.4 \mathrm{keV})$, the intermediate regime $(0.5-0.9 \mathrm{keV})$, and the hard X-ray band $(0.9-$ $2.0 \mathrm{keV})$. The $0.9-2.0 \mathrm{keV}$ band is clearly dominated by the nuclear source. The lower contours do not coincide with the bar's S-shape and are rather caused by two unresolved point sources in the spiral arms, one to the north, the other one to the northeast, both separated from the center by $1^{\prime}$. Both contour extensions coincide clearly with $\mathrm{HII}$ regions from the $\mathrm{H} \alpha$ image, the northern part with the luminous HII region in the disk of NGC 2903, indicating possible contribution from unresolved hard point sources, i.e. young SNRs and/or high-mass $\mathrm{X}$-ray binaries. In the intermediate-energy band $(0.5-0.9 \mathrm{keV})$ the X-ray emission covers the entire inner $4^{\prime}$ of the disk and traces quite good the most prominent HII emission regions. The northern point source visible in the HRI image located at the HII region in the northern spiral arm is also present in the PSPC exposure.

The structure in the soft energy image $(0.1-0.4 \mathrm{keV}$, Fig. 3b) is of particular interest, because of the distinct 

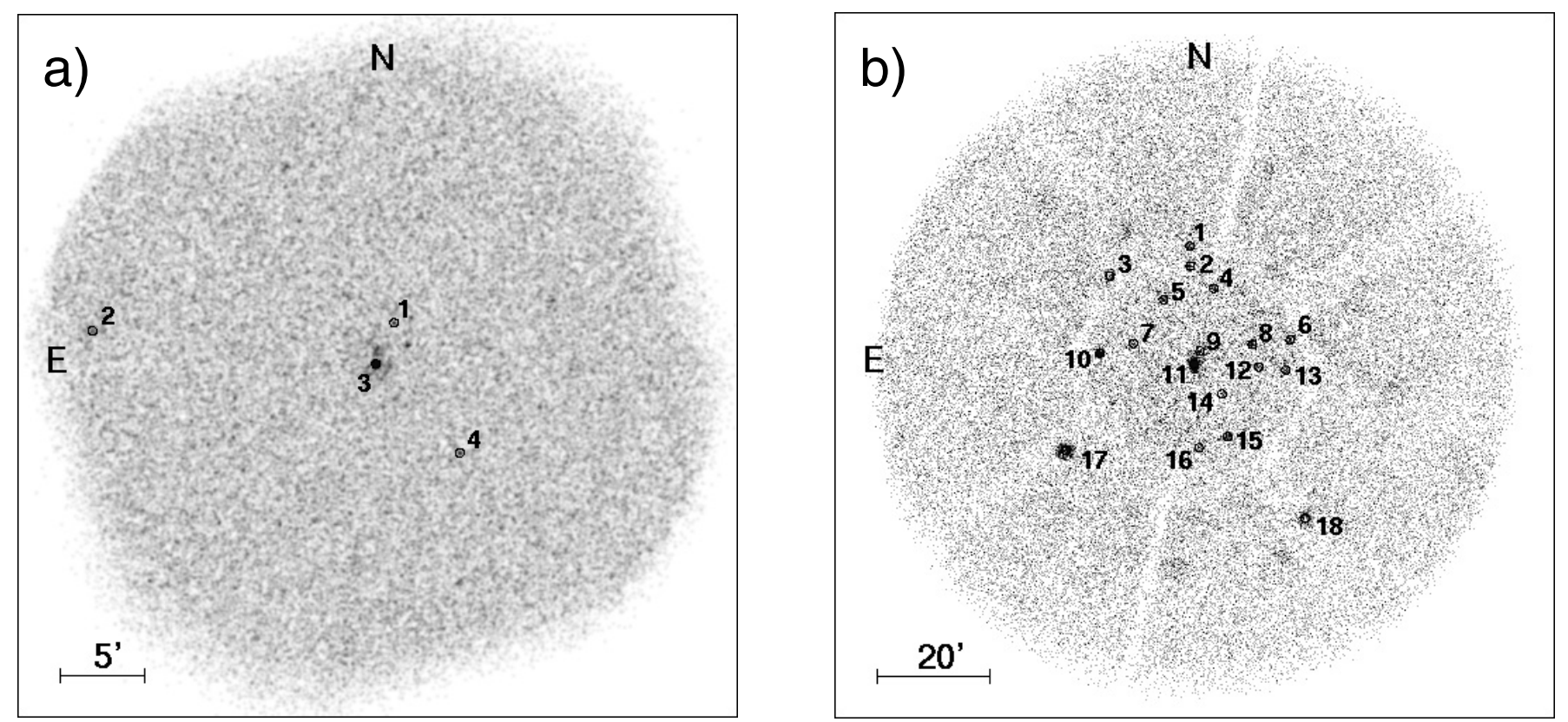

Fig. 1. Source detections in the ROSAT HRI a) and PSPC b) observation of NGC 2903 with a maximum likelihood threshold of 14 (5 $\sigma)$. The source numbers are listed in Table 3 (HRI) and Table 4 (PSPC), respectively.

extension in western direction perpendicular to the major axis. It significantly exceeds the inner spiral structure in $\mathrm{H} \alpha$ and the $0.5-0.9 \mathrm{keV}$ emission from the disk. At the given distance of 8.9 Mpc for NGC 2903 the projected radius of this soft feature is $5.2 \mathrm{kpc}$ from the center. We will discuss this feature in detail in Sects. 4.2 to 4.4. In the soft energy band the luminous HII region $1^{\prime}$ north of the central source, as detected in $\mathrm{H} \alpha$ and the hard PSPC band, also appears. In addition, it is clearly detectable by the HRI (Fig. 3).

No diffuse disk component in NGC 2903 is discernible in the HRI image. Here the most prominent source is again the nuclear region, covering the area of the optical hot spots. Its HRI and PSPC count rates of $(12.7 \pm 0.9) \times 10^{-3}$ cts s $^{-1}$ and $(36.9 \pm 2.2) \times 10^{-3}$ cts s $^{-1}$, respectively, are in good agreement, considering a 3 times smaller sensitivity for the HRI. The resolution of about $5^{\prime \prime}$ is not sufficient to clearly separate any single source within the innermost disk. Nevertheless, the X-ray emission covers an area of about $40^{\prime \prime} \times 30^{\prime \prime}$ at the $3 \sigma$ level contours. The third luminous X-ray source in the HRI image is the point source at the position of northern separate bright HII region at a distance of 2.7 from the center. This source appears about 1.5 times fainter in the HRI than in the PSPC, indicating a possible variability. But with the small count statistics of 14 counts in the HRI exposure this is hard to verify.

\subsection{Spectral distribution}

As can be seen from Fig. 2, different energy distributions emerge from the different components of the galaxy. Therefore we subdivide the PSPC image in several regions (see Fig. 4), namely, entire galaxy (area 1), nucleus (area 2), disk (areas 3a and $3 b$ ), eastern soft component (area 4), and northern prominent point source (area 5). A quantitative conclusion of the spectral distribution for the different areas can be drawn from
Table 5. Count rates and hardness ratios of the X-ray components of NGC 2903 as labeled in Fig. 4.

\begin{tabular}{lrr}
\hline \hline Component & $\begin{array}{l}\text { Count rate } \\
{\left[10^{-3} \mathrm{cts} \mathrm{s}^{-1}\right]}\end{array}$ & Hardness ratio \\
\hline 1 & $84.0 \pm 3.4$ & $+0.56 \pm 0.04$ \\
2 (nucleus) & $36.9 \pm 2.2$ & $+0.69 \pm 0.04$ \\
3a (northern disk) & $11.7 \pm 1.2$ & $+0.39 \pm 0.10$ \\
3b (southern disk) & $10.0 \pm 1.2$ & $+0.63 \pm 0.10$ \\
4 (halo) & $5.9 \pm 0.8$ & $-0.16 \pm 0.13$ \\
5 (point source) & $3.5 \pm 0.6$ & $+0.60 \pm 0.18$ \\
\hline
\end{tabular}

${ }^{a}$ Background subtracted.

the hardness ratio $H R$, defined by $(B-A) /(B+A)$, where $A$ and $B$ are the numbers of counts in the energy channels 11-41 $(0.1-0.4 \mathrm{keV})$ and 52-201 (0.5-2.0 keV), respectively. HR of the different components from Fig. 4 together with their count rates are listed in Table 5. Strikingly the $H R=-0.16$ of component No. 4 is the only negative value and differs strongly from the other regions which have values between +0.6 and +0.7 , i.e. reveal much harder X-ray spectra. Region No. 3a (northern disk) is with $H R=+0.39$ slightly softer than region No. $3 b$. This can already be perceived in Fig. 2. The bright HII region $1^{\prime}$ north of the center emits a significant fraction in the soft range. Nevertheless, the flux from this region is still quite hard compared to area 4.

In Fig. 5, the 0.1-2.4 keV spectra of the components as labeled in Fig. 4 are plotted. We fit several emission models to the spectra of the entire galaxy (area 1) and of the nucleus (area 2), like a power-law model (PO), a Raymond-Smith model (Raymond \& Smith 1977) for a hot plasma (RS), and a thermal Bremsstrahlung model (BS). For area 1 we also compare a combined $\mathrm{PO}+\mathrm{RS}$ model and a two-temperature $\mathrm{RS}+\mathrm{RS}$ 

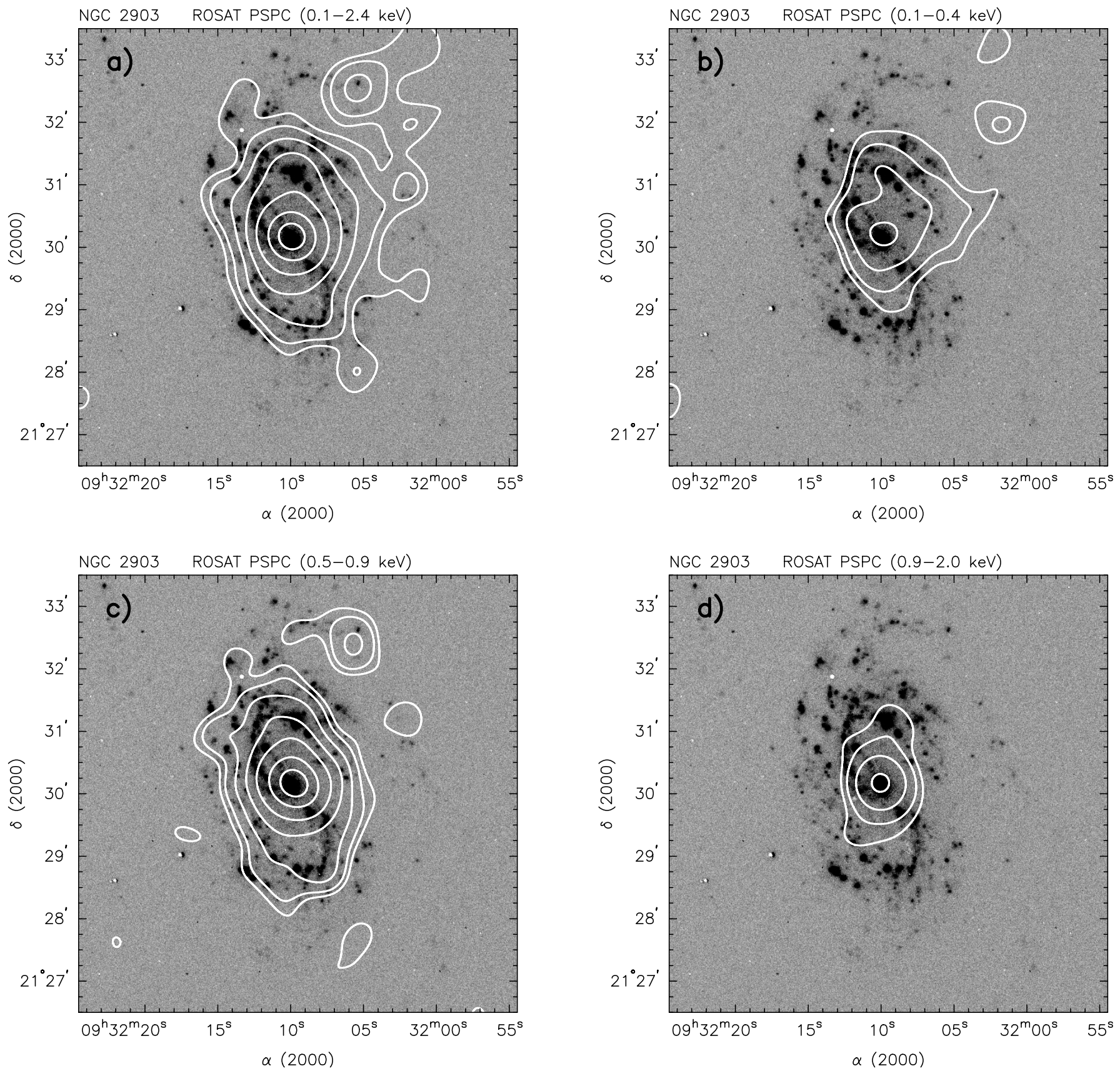

Fig. 2. ROSAT PSPC contours on top of the H $\alpha$ image (Boselli, private communication) of NGC 2903 in four different energy ranges: a) full PSPC bandwidth of $0.1-2.4 \mathrm{keV}$, b) $0.1-0.4 \mathrm{keV}$, c) $0.5-0.9 \mathrm{keV}$, d) $0.9-2.0 \mathrm{keV}$. Contour levels in each image are $(0.7,1.0,1.6,2.9,5.4,10.4$, $15.4) \times 10^{-6} \mathrm{cts} \mathrm{s}^{-1} \operatorname{arcsec}^{-2}$, corresponding to $3,5,10,20,40,80$, and $120 \sigma$, respectively.

model considering a high temperature central starburst component and a low temperature halo component. The results are listed in Tables 6 and 7.

The quality of the spectral distribution fits for the entire galaxy does not allow to prefer any of the chosen models. A single-temperature RS model with solar abundances, however, fits the spectrum obviously the worst but is improved for $1 / 10 Z_{\odot}$. This leads to the suggestion that the X-ray emitting plasma has low metallicity. An even better fit is achieved with a BS model (see Fig. 6). The temperature lies in the range of $0.7-1.0 \mathrm{keV}$, which also has been observed in other central starburst galaxies (e.g. NGC 1365: Stevens et al. 1999;
NGC 2146: della Ceca et al. 1999), whereas the X-ray luminosity range between $1-2 \times 10^{40} \mathrm{erg} \mathrm{s}^{-1}$.

Non-thermal models differ from the purely thermal ones by resulting in a higher total $0.1-2.4 \mathrm{keV}$ luminosity in the range of $2.8-4.5 \times 10^{40} \mathrm{erg} \mathrm{s}^{-1}$, The HI column density for these PO models lies at $6 \times 10^{20} \mathrm{~cm}^{-2}$, i.e. a factor of two higher than the Galactic foreground value of $2.8 \times 10^{20} \mathrm{~cm}^{-2}$ (Dickey \& Lockman 1990). In the RS+PO model the nonthermal component dominates the total flux by $90-95 \%$. So these cases require an explanation for such a high contribution by non-thermal sources, i.e. X-ray binaries, SNRs, and/or a central accretion-powered nucleus. Nevertheless, other 

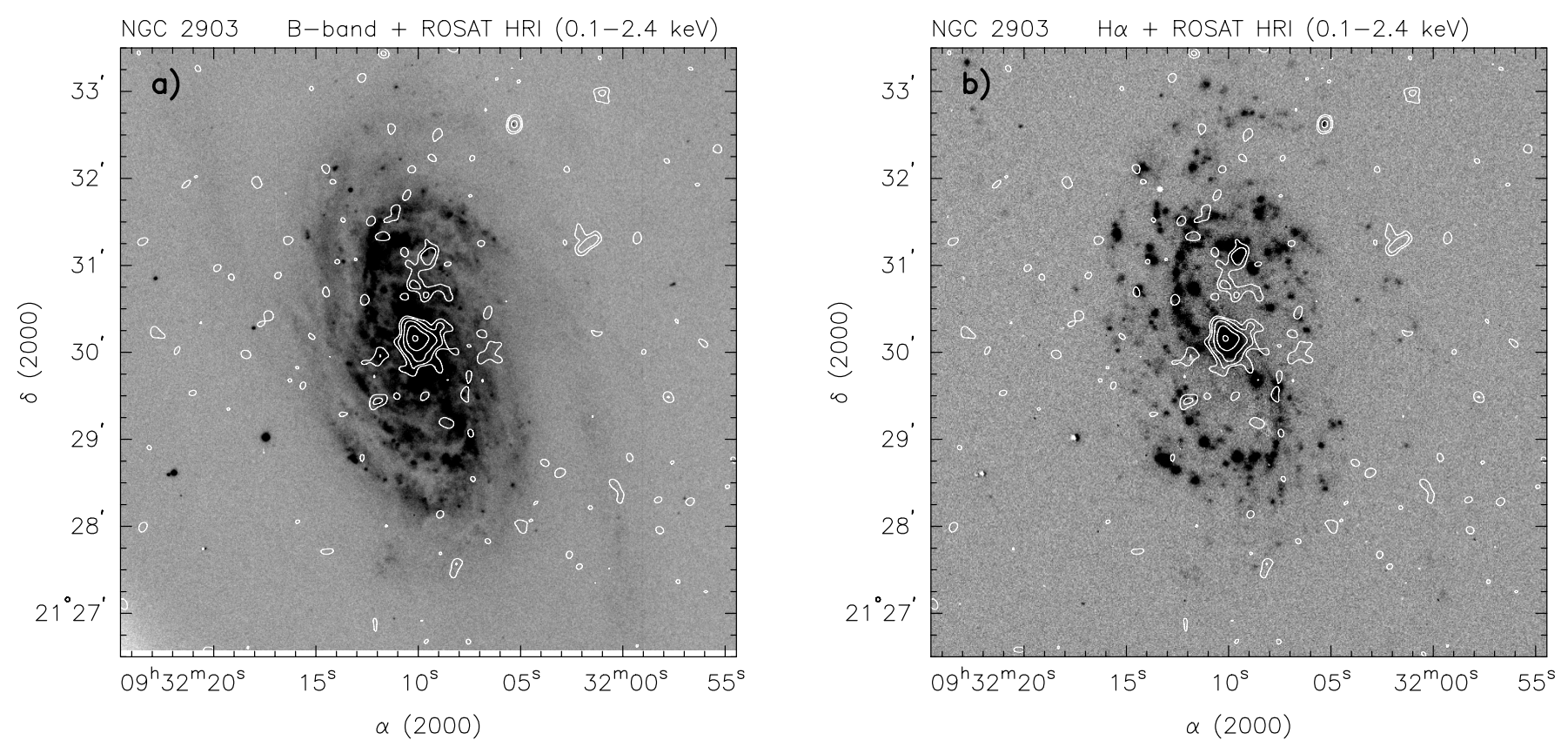

Fig. 3. ROSAT HRI contours over a) $B$-band and b) $\mathrm{H} \alpha$ image of NGC 2903. Contour levels are $(1.0,1.4,2.5,4.7,9.0) \times 10^{-6} \mathrm{cts} \mathrm{s}^{-1} \mathrm{arcsec}^{-2}$, corresponding to $3,5,10,20$, and $40 \sigma$, respectively.

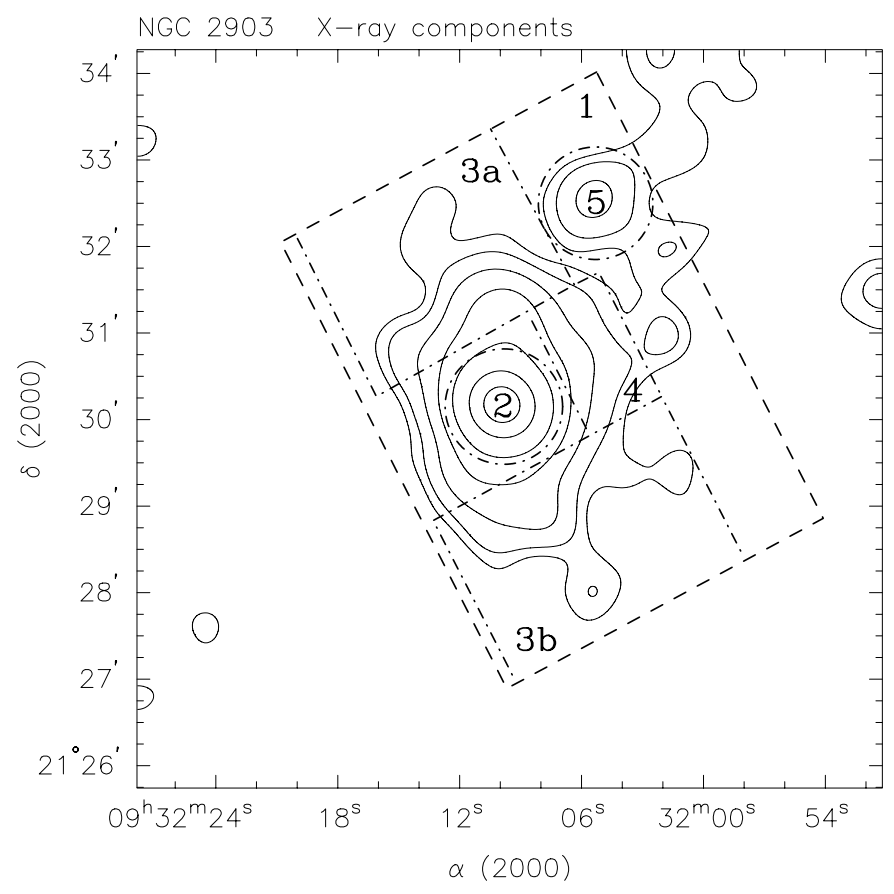

Fig. 4. Definition of areas for the different galaxy components in the PSPC exposure as used in the text: total galaxy (1) included by the dashed rectangle; nuclear region (2), northern disk (3a), southern disk (3b), halo (4), and northern point source (5) each marked by the dashdotted lines.

observations do not fulfill the general view of the galaxy center as an AGN. This agrees with our results from the nuclear component which also do not match the assumption of an AGN (see below).

In order to study a separate presumably low-temperature halo component (area 4, see Sect. 4.2) we also fit the spectrum of NGC 2903 with a two-component RS+RS model, as observed in edge-on starburst galaxies with hot extraplanar gas (DWH98). As a first approach, the abundances are fixed to solar, by this, representing the central starburst with its freshly synthesized material not yet mixed with the ambient ISM, and to $0.1 Z_{\odot}$ for the outflowing mixed plasma and the shock-heated swept-up ambient medium. The column density of the best fit lies in the range of the Galactic foreground value, and the plasma temperature for the low-metallicity component is with $0.3 \mathrm{keV}$ compatible with other observed X-ray halos (DWH98). Nevertheless, the luminosity fraction of $50 \%$ from such a soft halo component is unexpectedly high. In addition, an acceptable fit is only achieved for the solar metallicity component with a very high plasma temperature of $4 \mathrm{keV}$. This is not in agreement with ASCA observations analyzed by DWH98. On the other hand, the soft energy range of ROSAT is not well suited to verify such a hard component.

As a most reasonable model (last one in Table 6) at least we account for the different measured Hi column densities toward the different components RS+PO, respectively. For NGC 2903 the intrinsic column density (Wevers et al. 1986) ranges from at least $8 \times 10^{20} \mathrm{~cm}^{-2}$ in the inner disk (in particular the region covered by $\mathrm{X}$-ray emission) to a maximum of about $1.7 \times 10^{21} \mathrm{~cm}^{-1}$. Assuming that the PO contribution to the whole spectrum stems from the nucleus that is embedded into the HI, we apply as an averaged value nearly $7 \times 10^{20} \mathrm{~cm}^{-1}$ $\left(4 \times 10^{20} \mathrm{~cm}^{-1}\right.$ as half of the intrinsic disk plus $3 \times 10^{20} \mathrm{~cm}^{-1}$ for the galactic foreground) exceeding the disk component. Since the halo gas should be free of intrinsic extinction the RS component is only absorbed by means of the galactic foreground value of $2.8 \times 10^{20} \mathrm{~cm}^{-1}$.

The nuclear X-ray spectrum can better be explained by a thermal emission model at low-metallicity. Because of a decreasing contribution from emission lines in the model 

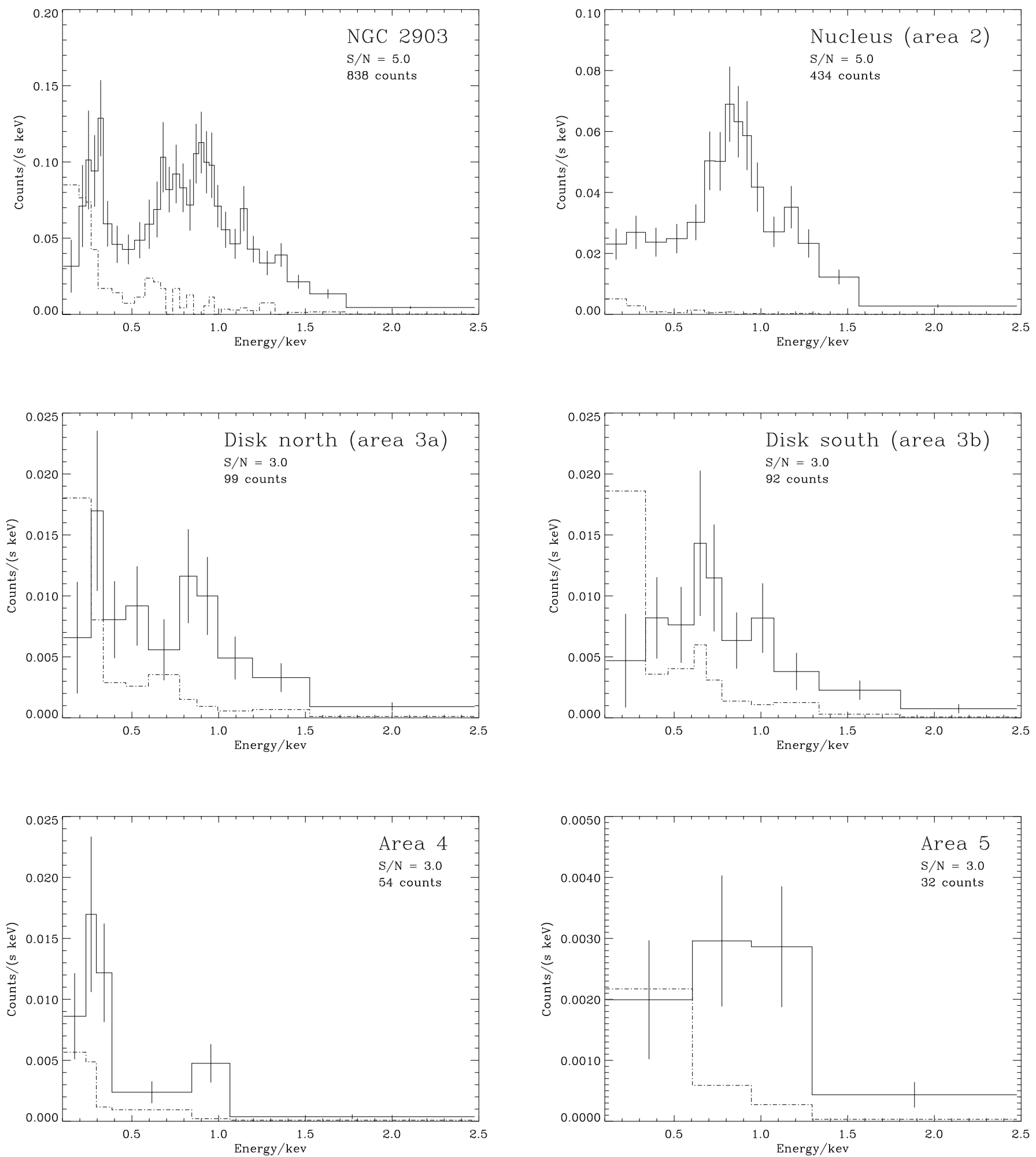

Fig. 5. Spectral background subtracted distribution (full drawn) of the different components (Fig. 4) in the $0.1-2.4$ keV energy range: a) total galaxy, area 1; b) nuclear region, area 2; c) northern disk, area 3a; d) southern disk, area 3b; e) halo, area 4; f) northern point source, area 5. For the total galaxy and the nucleus the spectra were binned to a signal-to-noise ratio of 5 , all other spectra have a ratio of 3 . The dash-dotted lines represent the background in each region.

sequence RS $\left(1 Z_{\odot}\right)$, RS $\left(0.1 Z_{\odot}\right)$, BS consequently the fraction of absorbed flux increases. This, however, rules out the solar metallicity RS model because the corresponding column density reaches only $9.5 \times 10^{19} \mathrm{~cm}^{-2}$ and by this lies far below the Galactic foreground value of $2.8 \times 10^{20} \mathrm{~cm}^{-2}$ and the intrinsic HI measurement by Wevers et al. (1986). For a $0.1 Z_{\odot}$ RS model the column density is somewhat higher $\left(3.5 \times 10^{20} \mathrm{~cm}^{-2}\right)$, while the BS model reaches $6.6 \times 10^{20} \mathrm{~cm}^{-2}$. 
Table 6. Fit results on the X-ray spectrum from NGC 2903 (area 1).

\begin{tabular}{|c|c|c|c|c|c|c|c|c|c|}
\hline $\begin{array}{l}\text { Model } \\
(1)\end{array}$ & $\begin{array}{l}N_{\mathrm{H}} \\
(2)\end{array}$ & $\begin{array}{l}k T \\
(3)\end{array}$ & $\begin{array}{l}\Gamma \\
(4)\end{array}$ & $\begin{array}{l}Z \\
(5)\end{array}$ & $\begin{array}{l}\text { Norm } \\
(6)\end{array}$ & $\begin{array}{l}\text { Red. } \chi^{2} \\
\text { (7) }\end{array}$ & $\begin{array}{l}\text { d.o.f. } \\
\text { (8) }\end{array}$ & $\begin{array}{l}F_{\mathrm{X}} \\
(9)\end{array}$ & $\begin{array}{l}L_{X} \\
(10)\end{array}$ \\
\hline RS & $0.50_{-0.13}^{+0.16}$ & $0.88_{-0.04}^{+\infty}$ & & 1 & 2.7 & 3.8 & 27 & $0.78_{-0.78}^{+0.07}$ & $0.74_{-0.74}^{+0.07}$ \\
\hline RS & $2.26_{-0.23}^{+0.26}$ & $1.01_{-0.08}^{+0.07}$ & & 0.1 & 16 & 1.4 & 27 & $1.31 \pm 0.01$ & $1.24 \pm 0.01$ \\
\hline BS & $3.74_{-0.45}^{+0.50}$ & $0.74_{-0.10}^{+0.11}$ & & & 3.3 & 1.0 & 27 & $1.90_{-0.14}^{+0.16}$ & $1.80_{-0.13}^{+0.15}$ \\
\hline $\mathrm{PO}$ & $6.01_{-0.64}^{+0.78}$ & & $2.81 \pm 0.17$ & & 4.0 & 1.0 & 27 & $4.79_{-0.94}^{+1.25}$ & $4.54_{-0.89}^{+1.18}$ \\
\hline $\mathrm{RS}+\mathrm{PO}$ & $4.91_{-0.92}^{+1.08}$ & $0.57_{-0.21}^{+0.25}$ & $2.61_{-0.16}^{+0.26}$ & 1 & $\begin{array}{l}3.3(\mathrm{PO}) \\
0.38(\mathrm{RS})\end{array}$ & 0.9 & 25 & $\begin{array}{l}3.16_{-0.52}^{+1.21} \\
(0.15 \pm 0.03)\end{array}$ & $\begin{array}{l}3.00_{-0.49}^{+1.15} \\
(0.14 \pm 0.03)\end{array}$ \\
\hline $\mathrm{RS}+\mathrm{PO}$ & $4.77_{-0.77}^{+0.96}$ & $0.55_{-0.21}^{+0.29}$ & $2.56 \pm 0.37$ & 0.1 & $\begin{array}{l}3.1(\mathrm{PO}) \\
3.5(\mathrm{RS})\end{array}$ & 0.9 & 25 & $\begin{array}{l}2.95_{-0.91}^{+1.62} \\
\left(0.30_{-0.07}^{+0.01}\right)\end{array}$ & $\begin{array}{l}2.80_{-0.86}^{+1.54} \\
\left(0.28_{-0.07}^{+0.01}\right)\end{array}$ \\
\hline $\mathrm{RS}+\mathrm{RS}$ & $2.68_{-0.72}^{+0.82}$ & $\begin{array}{l}4.34_{-2.65}^{+\infty} \\
0.31_{-0.08}^{+0.17}\end{array}$ & & $\begin{array}{l}1 \\
0.1\end{array}$ & $\begin{array}{l}6.8 \\
12\end{array}$ & 1.1 & 25 & $\begin{array}{l}1.50_{-0.82}^{+0.42} \\
\left(0.76_{-0.08}^{+0.21}\right)\end{array}$ & $\begin{array}{l}1.42_{-0.78}^{+0.40} \\
\left(0.72_{-0.08}^{+0.20}\right)\end{array}$ \\
\hline $1 \mathrm{~mm}] \mathrm{RS}+\mathrm{PO}$ & $\begin{array}{l}6.98(\mathrm{PO}, \text { fixed }) \\
2.82(\mathrm{RS}, \text { fixed })\end{array}$ & $0.87_{-0.86}^{+\infty}$ & $3.12_{-0.27}^{+0.47}$ & 0.1 & $\begin{array}{l}2.9(\mathrm{PO}) \\
4.5(\mathrm{RS})\end{array}$ & 1.0 & 26 & $\begin{array}{l}5.69_{-2.04}^{+6.03} \\
\left(0.40_{-0.39}^{+0.01}\right)\end{array}$ & $\begin{array}{l}5.39_{-1.93}^{+5.71} \\
\left(0.38_{-0.37}^{+0.01}\right)\end{array}$ \\
\hline
\end{tabular}

Column (1): spectral models: BS = thermal Bremsstrahlung, RS = Raymond-Smith, $\mathrm{PO}=$ power law.

Column (2): column density in units of $10^{20} \mathrm{~cm}^{-2}$.

Column (3): plasma temperature in units of $\mathrm{keV}$.

Column (4): photon index.

Column (5): metallicity in units of $Z_{\odot}$; fixed values.

Column (6): scaling factor: for BS in units of $\left(10^{-18} /\left(4 \pi D^{2}\right)\right) \int n_{\mathrm{e}} n_{\mathrm{I}} \mathrm{d} V, n_{\mathrm{e}}, n_{\mathrm{I}}=$ electron and ion densities $\left(\mathrm{cm}^{-3}\right)$; for RS in units of $\left(10^{-18} /\left(4 \pi D^{2}\right)\right) \int n_{\mathrm{e}}^{2} \mathrm{~d} V, n_{\mathrm{e}}, n_{\mathrm{H}}=$ electron and $\mathrm{HI}$ density $\left(\mathrm{cm}^{-3}\right)$; for PO in units of $10^{-4}$ photons $\mathrm{keV}^{-1} \mathrm{~cm}^{-2} \mathrm{~s}^{-1}$ at $1 \mathrm{keV}$.

Column (7): reduced $\chi^{2}$.

Column (8): degrees of freedom.

Column (9): unabsorbed X-ray flux in units of $10^{-12} \mathrm{erg} \mathrm{cm}^{-2} \mathrm{~s}^{-1}$. Values in brackets give the contribution of the thermal component.

Column (10): X-ray luminosity in units of $10^{40} \mathrm{erg} \mathrm{s}^{-1}$. Values in brackets give the contribution of the thermal component. For the RS+RS model the values in brackets are for the low-temperature component.

Table 7. Fit results on the X-ray spectrum from the nucleus of NGC 2903 (area 2).

\begin{tabular}{llllllllll}
\hline \hline $\begin{array}{l}\text { Model } \\
(1)\end{array}$ & $\begin{array}{l}N_{\mathrm{H}} \\
(2)\end{array}$ & $\begin{array}{l}k T \\
(3)\end{array}$ & $\begin{array}{l}\Gamma \\
(4)\end{array}$ & $\begin{array}{l}Z \\
(5)\end{array}$ & $\begin{array}{l}\text { Norm } \\
(6)\end{array}$ & $\begin{array}{l}\text { Red. } \chi^{2} \\
(7)\end{array}$ & $\begin{array}{l}\text { d.o.f. } \\
(8)\end{array}$ & $\begin{array}{l}F_{\mathrm{X}} \\
(9)\end{array}$ & $\begin{array}{l}L_{\mathrm{X}} \\
(10)\end{array}$ \\
\hline $\mathrm{RS}$ & $0.95_{-0.31}^{+0.42}$ & $0.82_{-0.07}^{+0.05}$ & & 1 & 1.1 & 2.7 & 9 & $0.36_{-0.03}^{+0.04}$ & $0.34_{-0.03}^{+0.04}$ \\
$\mathrm{RS}$ & $3.51_{-0.49}^{+0.62}$ & $0.78_{-0.10}^{+0.07}$ & & 0.1 & 6.6 & 1.2 & 9 & $0.59_{-0.01}^{+0.01}$ & $0.56_{-0.01}^{+0.01}$ \\
$\mathrm{BS}$ & $6.56_{-0.92}^{+1.31}$ & $0.54_{-0.10}^{+0.11}$ & & & 2.4 & 1.1 & 9 & $1.18_{-0.16}^{+0.14}$ & $1.12_{-0.15}^{+0.13}$ \\
PO & $9.71_{-1.00}^{+1.88}$ & & $3.27_{-0.25}^{+0.30}$ & & 2.0 & 1.4 & 9 & $4.70_{-1.50}^{+3.00}$ & $4.50_{-1.40}^{+2.90}$ \\
\hline
\end{tabular}

Column (1): spectral models: BS = thermal Bremsstrahlung, RS = Raymond-Smith, PO = power law.

Column (2): column density in units of $10^{20} \mathrm{~cm}^{-2}$.

Column (3): plasma temperature in units of $\mathrm{keV}$.

Column (4): photon index.

Column (5): metallicity in units of $Z_{\odot}$; fixed values.

Column (6): scaling factor: for BS in units of $\left(10^{-18} /\left(4 \pi D^{2}\right)\right) \int n_{\mathrm{e}} n_{\mathrm{I}} \mathrm{d} V, n_{\mathrm{e}}, n_{\mathrm{H}}=$ electron and ion densities $\left(\mathrm{cm}^{-3}\right)$; for RS in units of $\left(10^{-18} /\left(4 \pi D^{2}\right)\right) \int n_{\mathrm{e}} n_{\mathrm{H}} \mathrm{d} V, n_{\mathrm{e}}, n_{\mathrm{H}}=$ electron and $\mathrm{H}$ densities $\left(\mathrm{cm}^{-3}\right)$; for PO in units of $10^{-4}$ photons $\mathrm{keV}^{-1} \mathrm{~cm}^{-2} \mathrm{~s}^{-1}$ at $1 \mathrm{keV}$.

Column (7): reduced $\chi^{2}$.

Column (8): degrees of freedom.

Column (9): unabsorbed X-ray flux in units of $10^{-12} \mathrm{erg} \mathrm{cm}^{-2} \mathrm{~s}^{-1}$.

Column (10): X-ray luminosity in units of $10^{40} \mathrm{erg} \mathrm{s}^{-1}$.

Considering the source to be located in the central plane of the disk, this absorbing column density for the BS model is in agreement with a Galactic value of $2.8 \times 10^{20} \mathrm{~cm}^{-2}$ and half of the total intrinsic value of the HI disk of $8 \times 10^{20} \mathrm{~cm}^{-2}$, respectively.
The influence of emission lines in the spectrum is also perceptible in the nuclear luminosity because of the higher continuum flux for models with lower metallicities. The RS and BS fits for $0.1 Z_{\odot}$ result in $L_{X}=5.9 \times 10^{39} \mathrm{erg} \mathrm{s}^{-1}$ and $L_{\mathrm{X}}=1.2 \times 10^{40} \mathrm{erg} \mathrm{s}^{-1}$, respectively. Figure 7 shows the fits 

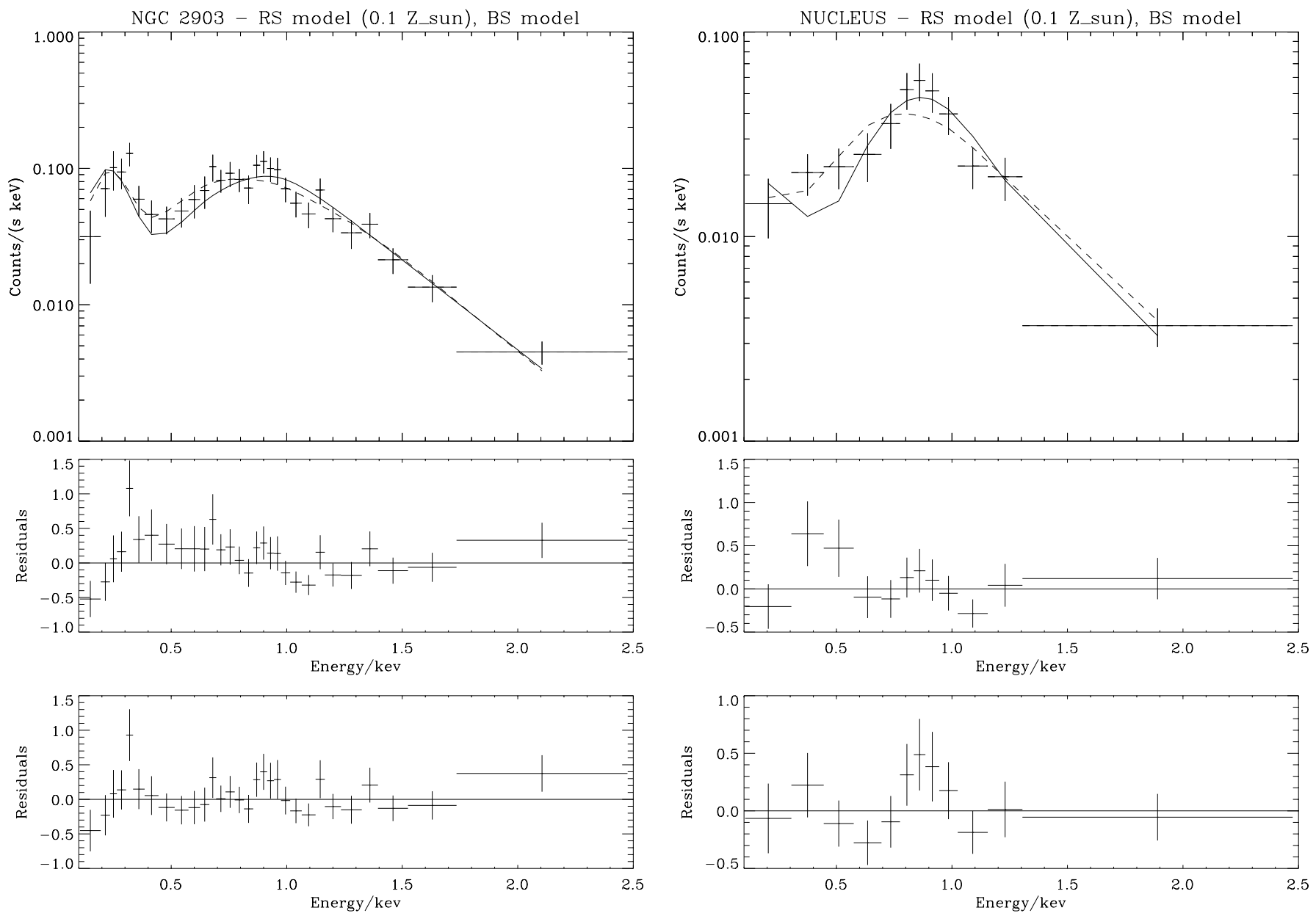

Fig. 6. Fits of a $0.1 Z_{\odot}$ Raymond-Smith model (full line, upper residuals) and a Bremsstrahlung model (dashed line, lower residuals) to the 0.1-2.4 keV spectrum of NGC 2903. Fit parameters are listed in Table 6.

of the RS $\left(0.1 Z_{\odot}\right)$ and the BS model. To achieve a good fit of the nuclear spectrum with a PO model a column density of about $10^{21} \mathrm{~cm}^{-2}$ is required. As a consequence the X-ray luminosity amounts to $4.5 \times 10^{40} \mathrm{erg} \mathrm{s}^{-1}$. This is much too high compared to the derived total X-ray luminosity of the entire galaxy in any of the applied models. Only in the case of a single PO model the nuclear luminosity would be as high as the total luminosity of NGC 2903 without explaining the contributions from the disk. For the other models the nuclear luminosity would even exceed the total luminosity (see Table 6). Because of the low count number it seems unreasonable to divide the emitting components into even further more, like DWH98 did it by $\mathrm{PO}+\mathrm{RS}+\mathrm{RS}$.

\section{Discussion}

\subsection{Nuclear and disk X-ray emission - star formation rate}

Comparing the X-ray images from both ROSAT detectors we find that the diffuse disk emission in the low-resolution PSPC image has not been detected with the HRI. Only the strongest disk sources (north and southeast from the center) have

Fig. 7. Model fit of the $0.1-2.4 \mathrm{keV}$ spectrum of the nucleus (area 2) a $0.1 Z_{\odot}$ Raymond-Smith model (full line, upper residuals) and a Bremsstrahlung model (dashed line, lower residuals). Fit parameters are listed in Table 7.

counterparts in both HRI and PSPC images (most easily visible in the hard band, Fig. 2d). On the other hand, if we notice that the HRI detector is about three times less sensitive compared to the PSPC detector, sources at count rates lower than the strongest disk sources cannot be distinguished from the background in the HRI exposure. Depending on the emission model, the contribution of the disk to the total luminosity amounts to $40-50 \%$.

According to Leitherer (1994), the X-rays from SF regions are dominated by supernovae after $10^{7}$ years. To determine the mean SF rate $\Psi$ the disk of NGC 2903 one can use the simplistic approach from Junkes et al. (1995) based on the work by Cioffi (1990) for single cooling supernova shells:

$\Psi\left[\mathrm{M}_{\odot} \mathrm{yr}^{-1}\right]=1.1 \times 10^{-40} L_{\mathrm{X}}\left[\mathrm{erg} \mathrm{s}^{-1}\right]$.

However, this approach gives a lower limit of $\Psi$ since it does not take into account any effect by accumulation and overlap of single supernova remnants. The SF rate for the total galaxy is thus derived from the best purely thermal (also RS+RS) models to $\Psi_{\mathrm{X}} \approx 1.4-2.0 M_{\odot} \mathrm{yr}^{-1}$. Corresponding to the results from the spectral distribution of nucleus and total galaxy about $50 \%$ of the total SF rate should take place in the nuclear region. 
Table 8. Star-formation rates for NGC 2903 derived from different spectral regimes.

\begin{tabular}{|c|c|c|c|}
\hline Spectral regime & $\begin{array}{l}\text { Luminosity } \\
{\left[\mathrm{erg} \mathrm{s}^{-1}\right]}\end{array}$ & $\begin{array}{l}\text { SF rate } \\
{\left[M_{\odot} \mathrm{yr}^{-1}\right]}\end{array}$ & Ref. $^{a}$ \\
\hline \multirow[t]{2}{*}{$\mathrm{H} \alpha$} & \multirow[t]{2}{*}{$2.5 \times 10^{41}$} & 2.2 & 1 \\
\hline & & 1.4 & 2 \\
\hline FIR & $3.4 \times 10^{43}$ & 5.7 & 3 \\
\hline $\mathrm{X}$-rays & $(1.2-1.8) \times 10^{40}$ & $1.4-2.0$ & 4 \\
\hline \multicolumn{4}{|c|}{ References: } \\
\hline \multicolumn{4}{|c|}{$\begin{array}{l}\text { I) Luminosity: Jackson et al. (1991); } \\
\text { IMF: Kennicutt (1983). }\end{array}$} \\
\hline \multicolumn{4}{|c|}{ ) Luminosity: Jackson et al (1991): } \\
\hline \multicolumn{4}{|c|}{ IMF: Pogge \& Eskridge (1987). } \\
\hline \multicolumn{4}{|c|}{ 3) Luminosity: Rice et al. (1988); } \\
\hline \multicolumn{4}{|c|}{$L_{\mathrm{FIR}}-\mathrm{SFR}$ relation: Thronson \& Telesco (1986). } \\
\hline \multicolumn{4}{|c|}{ Luminosity: this work; } \\
\hline
\end{tabular}

The SF rate can also be derived from the $\mathrm{H} \alpha$ emission of HII regions under the assumption of a specific IMF (Kennicutt 1983; Pogge \& Eskridge 1987). Jackson et al. (1991) measured the $\mathrm{H} \alpha$ flux resulting in a total luminosity of $2.5 \times 10^{41} \mathrm{erg} \mathrm{s}^{-1}$ (for a distance of $8.9 \mathrm{Mpc}$ ). Depending on the conversion law the derived $\Psi_{\mathrm{H} \alpha}$ amounts to $2.2 M_{\odot} \mathrm{yr}^{-1}$ (Kennicutt 1983) or $1.4 M_{\odot} \mathrm{yr}^{-1}$ (Pogge \& Eskridge 1987), respectively. About $13 \%$ of the total $\mathrm{H} \alpha$ flux originates from the hot-spot region of the galactic center, as mentioned by Jackson et al. (1991) and confirmed by the $\mathrm{H} \alpha$ data, provided by A. Boselli (private comm.). Because of the low spatial resolution of the PSPC the chosen boundaries for the nuclear region is somewhat larger than the hot spots resolved in $\mathrm{H} \alpha$ and, thus, area 2 contains also contributions from the inner disk (compare Figs. 2a and 4). This may cause the higher SF rate for the nucleus of about $0.5 M_{\odot} \mathrm{yr}^{-1}$ derived from the X-ray data.

In the FIR regime the total luminosity (for a distance of $8.9 \mathrm{Mpc}$ ) amounts to $8.8 \times 10^{9} L_{\odot}$ (Rice et al. 1988). By applying the $L_{\mathrm{FIR}}-\Psi$ relationship by Thronson \& Telesco (1986) the resulting $\mathrm{SF}$ rate $\Psi_{\mathrm{FIR}}$ differs significantly from the latter values. For the total galaxy we get $5.7 M_{\odot} \mathrm{yr}^{-1}$, while the nuclear region contributes $2.6 M_{\odot} \mathrm{yr}^{-1}$, if we use the estimation of $4 \times 10^{9} L_{\odot}$ for the central FIR luminosity from Telesco \& Harper (1980). This discrepancy may reflect the uncertainty by the simplified model to determine the SF rate from the X-ray flux. Since most of the soft X-ray emission originates from the thin bubble shell, the swept-up ambient material, and internal shocks (Heckman et al. 1990) the effect of accumulating bubbles may be essential. Thronson \& Telesco (1986) compared SFRs derived from $L_{\text {FIR }}$ with those from $\mathrm{H} \alpha$ luminosities pointing to uncertainties due to extinction. The discrepancy for NGC 2903 is well within the range of their result. The SF rates for NGC 2903 derived from different regimes are summarized in Table 8.

\subsection{The soft western halo component}

As already mentioned in Sect. 3.1 the main interesting feature in the PSPC data is the very soft $(H R=-0.16) \mathrm{X}$-ray emission in area 4 (Fig. 2b). Its lowest contour $(3 \sigma)$ extends to $2^{\prime}(5.2 \mathrm{kpc})$ from the nucleus to the west in the $0.1-0.4 \mathrm{keV}$ regime without any similar counterpart along the minor axis to the east. Comparison with Fig. $2 \mathrm{c}$ reveals that the asymmetrical structure exceeds the extension of the $0.5-0.9 \mathrm{keV}$ disk component and is only discernible in the soft regime, whereas the spatial distribution in the intermediate energy range follows the optical disk. Since the HI map of NGC 2903 (Wevers et al. 1986) gives a column density of at least $8 \times 10^{20} \mathrm{~cm}^{-2}$ in the inner disk (in particular the region covered by X-ray emission) with a maximum of about $1.7 \times 10^{21} \mathrm{~cm}^{-1}$, this soft extended component cannot originate from behind the galactic disk. Reasonably, the emission may stem from one or several giant HII regions in the disk as is the case for the northern HRI disk source. Whereas, however, the corresponding northern HII region is clearly detectable in the $\mathrm{H} \alpha$ image, no strong $\mathrm{H} \alpha$ emission is visible in the western part of the disk. Even if the soft component would originate from unresolved strong point sources they would also have to appear in the HRI image. A probable foreground star can be excluded because there is no corresponding object in the optical broad band image. We therefore exclude this soft emission component to originate from discrete sources. A diffuse source detectable at a $3 \sigma$ level in the HRI exposure would have a count rate of at least $1 \times 10^{-6} \mathrm{cts} \mathrm{s}^{-1} \operatorname{arcsec}^{-2}$. Comparing the count rate of $5.9 \times 10^{-3}$ cts s $^{-1}$ for area 4 in the PSPC exposure, taking an area size of $1.63 \mathrm{arcmin}^{2}$, and considering the lower detector sensitivity of the HRI detector, the expected count rate for this diffuse emission is about $3.3 \times 10^{-7} \mathrm{cts} \mathrm{s}^{-1} \operatorname{arcsec}^{-2}$. So it is not expected to be detectable in our HRI exposure.

In the next sections we will discuss the most plausible source for the soft emission with this morphology of a starburst galaxy, namely, the existence of a galactic wind and its interaction with the surrounding IGM.

\subsection{A galactic wind}

Since NGC 2903 houses a central starburst, an X-ray halo as observed in several edge-on galaxies (M 82: e.g. Lehnert et al. 1999; Weaver et al. 2000; NGC 253: e.g. Pietsch et al. 2000; NGC 2146: della Ceca et al. 1999; DWH98; NGC 3628: Dahlem et al. 1996; NGC 4565: Vogler et al. 1996; NGC 4569: Tschöke et al. 2001) can cause this observational feature. X-ray halos in actively star-forming galaxies are believed to be produced by giant kpc-scale outflows of hot gas from stellar winds and cumulative supernova explosions in the central starbursts (Tomisaka \& Ikeuchi 1988) forming a superbubble that expands into the ambient ISM, preferably perpendicular to the galactic disk with velocities up to several $1000 \mathrm{~km} \mathrm{~s}^{-1}$. Internal shocks of subsequent explosions heat up the bubble gas to some $10^{7}$ to $10^{8} \mathrm{~K}$ (Michaelis et al. 1996) and leads to hard (about $10 \mathrm{keV}$ ) X-ray emission. Evaporation of density inhomogeneities (clouds) in the halo and the adiabatic cooling due to expansion then decrease the temperature and, by this, soften the X-ray emission and and even lead to optical/UV line emission. Also the thin swept-up shell can cause strong 
optical line emission and soft X-rays (Heckman et al. 1993; Strickland \& Stevens 2000).

We already reported the existence of a similar X-ray feature in the Virgo Cluster galaxy NGC 4569 (Tschöke et al. 2001). In this object the soft western X-ray emission coincides with a huge $\mathrm{H} \alpha$ filament (Bomans et al. 2002). This remarkable feature is unique in the sense that a coincidence of $\mathrm{H} \alpha$ and X-ray emission of this extension has not been observed in comparable starburst or other edge-on galaxies so far. In the case of NGC 2903 there is no diffuse $\mathrm{H} \alpha$ emission connected with the soft X-ray region. Noticeably, NGC 2903 is not a cluster member and has no strongly perturbed morphology or nearby companion.

Under the assumption of a symmetrical bipolar outflow the absence of a corresponding eastern counterpart with the same extension as the western one supports the galactic disk orientation in space with the eastern part lying closer to the observer. Any X-ray emission from that eastern region would have to penetrate through the galactic disk with a column density of at least $8 \times 10^{20} \mathrm{~cm}^{-2}$. If the outflowing plasma has already cooled significantly, its soft X-ray flux is then fully blocked so that no detection is expected.

\subsection{Physical halo properties}

If we assume the western soft component to originate from a superwind propagating from the galactic center of NGC 2903 perpendicularly into the halo, we can derive from the observed $\mathrm{X}$-ray emission some physical parameters of the hot halo gas, like temperature $T$, mean electron density $n_{\mathrm{e}}$, mass $M$, and cooling time scale $\tau_{\text {cool }}$. Given the X-ray luminosity and the volume of the halo gas $V$ we can estimate the electron density from the emissivity of a thin hot plasma $\Lambda$ for a RS model by the equation $L_{\mathrm{X}}=n_{\mathrm{e}}^{2} \Lambda(T) V$ (see e.g. Bomans et al. 1997). The $\mathrm{X}$-ray flux from the halo region is quite faint. For a signal-tonoise ratio of 3 we get a spectrum of only 6 bins ( 54 counts, see Fig. 5e). So we have to presume some physical boundaries for the extraplanar gas. For the geometry we assume a conical shape, as sketched in Fig. 8, perpendicular to the disk with an inclination of $66^{\circ}$. Adapted from Fig. 4 for region No. 4 the characterizing dimensions are $R_{\max }=4.5 \mathrm{kpc}$ and $R_{\min }=3.1 \mathrm{kpc}$ and represent the distance of the galactic center to the northern corner of area 4 and the smallest distance of the galactic center to the western edge, respectively. $D_{\max }=4.1 \mathrm{kpc}$ corresponds to the NE-SW extension so that an opening cone angle of $54^{\circ}$ results. This results in an X-ray halo volume of $1.1 \times 10^{10} \mathrm{pc}^{3}$.

Contamination from nucleus and disk increases with smaller distance from the center. We furthermore assume a total HI column density for this region equal to the Galactic foreground value of $2.8 \times 10^{20} \mathrm{~cm}^{-2}$. The $H R$ of -0.16 for the ROSAT PSPC corresponds to a RS spectrum with a plasma temperature of about $0.2 \mathrm{keV}$. Under these conditions we derive a $0.1-2.4 \mathrm{keV}$ luminosity for the X-ray halo of $L_{\mathrm{X}} \approx$ $4 \times 10^{38} \mathrm{erg} \mathrm{s}^{-1}$. Applying a single RS model with $0.1 \mathrm{Z}_{\odot}$, this leads to an emissivity $\Lambda=4.8 \times 10^{-24} \mathrm{erg} \mathrm{s}^{-1} \mathrm{~cm}^{3}$ and results in a mean electron density for the extraplanar gas

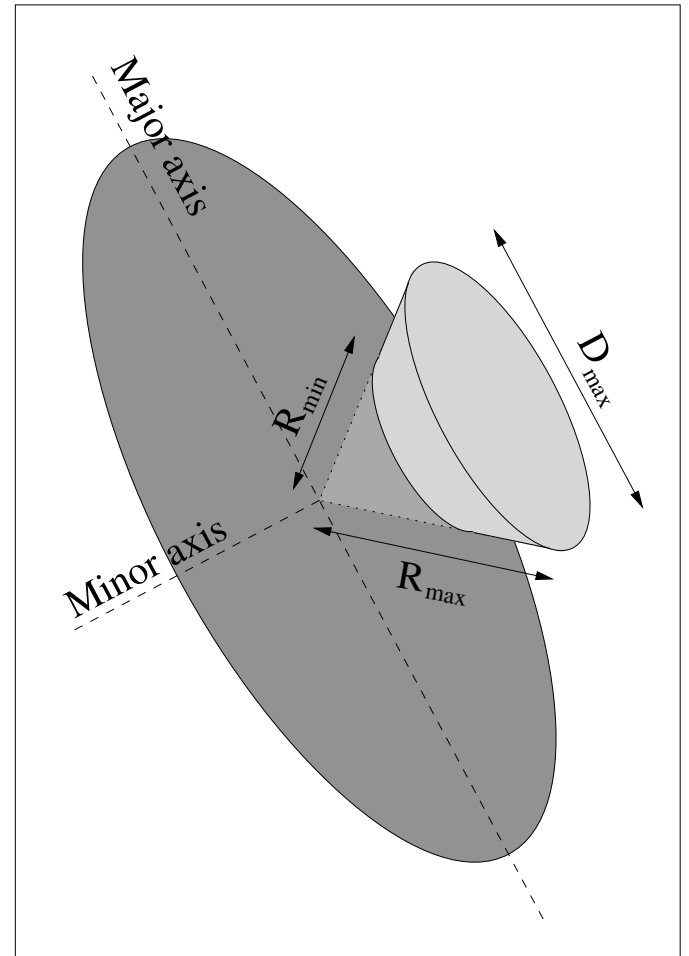

Fig. 8. Sketch of the applied geometry for the central outflow. The lightgray area margins the X-ray emitting volume corresponding region 4 in Fig. 4 . To determine $R_{\max }, R_{\min }$, and $D_{\max }$, an outflow orientation perpendicular to the disk with the inclination of $66^{\circ}$ has been used.

of $0.016 f^{-0.5} \mathrm{~cm}^{-3}$, depending on the volume filling factor $f$ of the X-ray emitting gas. The total mass amounts to $4.3 \times 10^{6} f^{0.5} M_{\odot}$. The cooling timescale $\tau_{\text {cool }}$ of the gas is $3 \mathrm{kT} / n \Lambda=3.8 \times 10^{8} f^{0.5} \mathrm{yrs}$ and the thermal energy and thermal pressure of the X-ray halo can be determined to $E_{\text {th }}=\frac{3}{2} n V k T=1.6 \times 10^{54} f^{0.5}$ erg and to $P=2 n k T=$ $9.7 \times 10^{-12} f^{-0.5} \mathrm{dyn} \mathrm{cm}^{-2}$, respectively.

The estimation of these properties is based on several less exact assumptions. To determine the reliability of the values for the halo gas we try to confine the most important error sources. The plasma temperature determined from the hardness ratio mostly depends on the chosen absorbing column density. It is much less sensitive with respect to metallicity. If we take as an upper limit of $8 \times 10^{20} \mathrm{~cm}^{-2}$ the value of the NGC 2903 HI disk, the uncertainty of the temperature is about $40 \%$ and that of the corresponding emissivity of the RS plasma about $20 \%$, respectively. $\Lambda$ is basically sensitive to the given metallicity. With solar metallicities the emissivity of the plasma at that temperature increases by a factor of 5 compared to $0.1 Z_{\odot}$. As a consequence, this results in a 0.4 times smaller electron density. From the error estimation of plasma temperature and absorbing component the resulting error for the $\mathrm{X}$-ray luminosity reaches $50 \%$. Therefore we estimate the uncertainty for the mean electron density to $7 \times 10^{-3} \mathrm{~cm}^{-3}$, not including the error from the metallicity in the determination of $\Lambda$ and the simplified geometry of the halo volume. The obtained physical parameters for the X-ray halo of NGC 2903 are listed in Table 9 for two different metallicities and two different volume filling factors. 
Table 9. Physical properties of the X-ray halo of NGC 2903 with different volume filling factors and metallicities of the X-ray emitting plasma.

\begin{tabular}{lllll}
\hline \hline Plasma metallicity $Z / Z_{\odot}$ & 0.1 & 0.1 & 1 & 1 \\
Volume filling factor $f$ & 1 & 0.2 & 1 & 0.2 \\
\hline Temperature $k T / \mathrm{keV}$ & $0.19_{-0.08}^{+0.07}$ & $0.19_{-0.08}^{+0.07}$ & $0.18_{-0.08}^{+0.07}$ & $0.18_{-0.08}^{+0.07}$ \\
Luminosity $L_{\mathrm{X}} /\left(10^{38} \mathrm{erg} \mathrm{s}^{-1}\right)$ & $4 \pm 2$ & $4 \pm 2$ & $4 \pm 2$ & $4 \pm 2$ \\
Electron density $n /\left(10^{-2} \mathrm{~cm}^{-3}\right)$ & $1.6 \pm 0.6$ & $3.6 \pm 1.3$ & $0.7 \pm 0.2$ & $1.6 \pm 0.6$ \\
X-ray halo mass $M /\left(10^{6} M_{\odot}\right)$ & $4.3 \pm 1.5$ & $1.9 \pm 0.6$ & $1.9 \pm 0.6$ & $0.8 \pm 0.2$ \\
Cooling time $\tau /\left(10^{8} \mathrm{yr}\right)$ & $3.8_{-2.1}^{+5.9}$ & $1.7_{-1.0}^{+2.7}$ & $1.6_{-0.9}^{+2.5}$ & $0.7_{-0.5}^{+1.2}$ \\
Thermal energy $E_{\mathrm{th}} /\left(10^{54} \mathrm{erg}\right)$ & $1.6_{-1.2}^{+1.6}$ & $0.7_{-0.5}^{+0.7}$ & $1.0_{-0.8}^{+1.0}$ & $0.4_{-0.3}^{+0.4}$ \\
Pressure $P /\left(10^{-12} \mathrm{dyn} \mathrm{cm}^{-2}\right)$ & $9.7 \pm 6.7$ & $22 \pm 15$ & $4.0 \pm 2.7$ & $8.9 \pm 6.2$ \\
\hline
\end{tabular}

Comparing the halo properties of NGC 2903 with other starbursting edge-on galaxies we find that the low X-ray temperature fits well with this group of galaxies with low plasma temperatures of the outflows in the range of $0.16-0.3 \mathrm{keV}$. The $0.1-2.4 \mathrm{keV}$ halo luminosity, however, is somewhat lower. This is plausibly understood by the fact that the luminosity of NGC 2903 is only derived from the outer part of the outflow (region 4) in order to derive uncontaminated halo properties. The cooling time scale of about $10^{8} \mathrm{yr}$ is high enough that the expanding plasma still emits in the X-rays at kpc-scale distances from the starburst.

\section{Conclusions}

The spatial and spectral distribution of the $0.1-2.4 \mathrm{keV} \mathrm{X}$ ray emission of the late-type 'hot-spot' galaxy NGC 2903 observed by ROSAT strongly suggests the existence of an extended X-ray gas component to about $5 \mathrm{kpc}$ above the disk of NGC 2903. This galaxy and NGC 4569 (Tschöke et al. 2001) are the only reported objects at intermediate inclination with an observed X-ray halo so far. In contrast to NGC 4569, where additionally an $\mathrm{H} \alpha$ filament as extended as the X-ray halo exists (Bomans et al. 2002) and the interaction with the ICM of the Virgo cluster plays an important role for the observed galaxy morphology, NGC 2903 is isolated without any perturbing companion. So the detected extraplanar X-ray component cannot be caused by external processes.

Its derived properties match with the observed X-ray halos in edge-on galaxies. From the spectral distribution we obtain a plasma temperature of $0.2 \mathrm{keV}$ and a luminosity of $4 \times 10^{38} \mathrm{erg} \mathrm{s}^{-1}$ as a lower limit because we cut out disk and nuclear regions for reasons of contamination. Therefore, the $\mathrm{X}$-ray luminosity may be higher at least by a factor of 2 . Mass and electron density of the X-ray emitting material in the considered region lie in the range of several $10^{6} M_{\odot}$ and $10^{-3} \mathrm{~cm}^{-3}$, respectively. A more accurate determination of the physical properties of the halo component requires deeper exposures, a higher spectral resolution for a reliable determination of the metallicity of the soft X-ray emitting gas, and a better spatial resolution of the outflow structure.

The spatial distribution of the $0.5-0.9 \mathrm{keV}$ emission coincides with the $\mathrm{H} \alpha$ image of NGC 2903 , indicating ongoing SF throughout the disk with a rate of $1-2 M_{\odot} \mathrm{yr}^{-1}$, consistent with the value derived from $\mathrm{H} \alpha$. The fraction of the SF rate from the nuclear region estimated by the X-ray flux is about $50 \%$.
This is somewhat higher than the value given by Jackson et al. (1991) who estimated about $13 \%$ of the $\mathrm{H} \alpha$ flux contributed by the nuclear region. However, the low spatial resolution of the PSPC has to be taken into account. Because of the simplified model the resulting values are possibly by a factor of $2-5$ too low as the FIR emission in NGC 2903 lets suggest.

Acknowledgements. The authors are grateful to Alessandro Boselli for stimulating discussions, and kindly providing the $\mathrm{H} \alpha$ data, and to Polichronis Papaderos for providing the optical broadband image. The paper benefits from constructive remarks by an unknown referee. The ROSAT project is supported by the German Bundesministerium für Bildung, Wissenschaft, Forschung und Technologie (BMBF) and the Max-Planck-Society. This research has made use of the NASA/IPAC Extragalactic Database (NED) which is operated by the Jet Propulsion Laboratory, Caltech, under contract with the NASA. D.T. was partly supported by the Deutsche Forschungsgemeinschaft under project no. He 1487/23-1.

\section{References}

Alonso-Herrero, A., Ryder, S. D., \& Knapen, J. H. 2001, MNRAS, 322,757

Arnaud, K. A. 1996, Astronomical Data Analysis Software and Systems V, ed. G. Jacoby, \& J. Barnes, ASP Conf. Ser., 101, 17

Bomans, D. J., Chu, Y.-H., \& Hopp, U. 1997, AJ, 113, 1678

Bomans, D. J., Tschöke, D., Hensler, G., et al. 2002, A\&A, submitted Briel, U., Aschenbach, B., Hasinger, G., et al. 1996, ROSAT User's Handbook, MPE, Garching

Cioffi, D. 1990, in Physical Processes in Hot Plasmas, ed. W. Brinkmann, A. C. Fabian, \& F. Giovanelli (Dordrecht: Kluwer), NATO ASI Ser. C, 305, 1

Dahlem, M., Heckman, T. M., Fabbiano, G., Lehnert, M. D., \& Gilmore, D. 1996, ApJ, 461, 724

Dahlem, M., Weaver, K. A., \& Heckman, T. M. 1998, ApJS, 118, 401 (DWH98)

della Ceca, R., Griffiths, R. E., Heckman, T. M., Lehnert, M. D., \& Weaver, K. A. 1999, ApJ, 514, 772

Dickey, J. M., \& Lockman, F. J. 1990, ARA\&A, 28, 215

Drozdovsky, I. O., \& Karachentsev, I. D. 2000, A\&AS, 142, 425

Falco E., Kurtz M., Gellar M., et al. 1999, PASP, 111, 438

Freyer, T., \& Hensler, G. 2000, Astrophysical Plasmas: Codes, Models, and Observations, ed. J. Arthur, N. Brickhouse, \& J. Franco, Rev. Mex. Astron. Astrofis. Conf. Ser., 9, 187

Heckman, T. M., Armus, L., \& Miley, G. K. 1990, ApJS, 74, 833

Heckman, T. M., Lehnert, M. D., \& Armus, L. 1993, in The Environment and Evolution of Galaxies, ed. J. M. Shull, \& H. A. Thronson (Dordrecht: Kluwer), 455

Jackson, J. M., Eckart, A., Cameron, M., et al. 1991, ApJ, 375, 105 
Junkes, N., Zinnecker, H., Hensler, G., Dahlem, M., \& Pietsch, W. 1995, A\&A, 294, 8

Kennicutt, R. C., Jr. 1983, ApJ, 272, 54

Laques, P., Nieto, J.-L., Vidal, J.-L., Augé, A., \& Despiau, R. 1980, Nature, 288, 145

Lehnert, M. D., Heckman, T. M., \& Weaver, K. A. 1999, ApJ, 523, 575

Leitherer, C. 1994, Rev. Mod. Astr., 7, 73

Michaelis, O., Hensler, G., Samland, M. 1996, Proc. 11th IAP Meeting, The Interplay between Massive Star Formation, the ISM and Galaxy Evolution, ed. D. Kunth (Gif-sur-Yvette: Éditions Frontières), 525

Norman, C. A., \& Ikeuchi, S. 1989, ApJ, 345, 372

Oka, S., Wakamatsu, K., \& Sakka, K. 1974, PASJ, 26, 289

Pietsch, W., Vogler, A., Klein, U., \& Zinnecker, H. 2000, A\&A, 360, 24

Planesas, P., Colina, L., \& Pérez-Olea, D. 1997, A\&A, 325, 81

Pogge, R. W., \& Eskridge, P. B. 1987, AJ, 93, 291

Raymond, J. C., \& Smith, B. W. 1977, ApJS, 35, 419

Rice, W., Lonsdale, C. J., Soifer, B. T., et al. 1988, ApJS, 68, 91

Shields, G. A. 1990, ARA\&A, 28, 525

Sérsic, J. L. 1973, PASP, 85, 103
Simons, D. A., DePoy, D. L., Becklin, E. E., et al. 1988, ApJ, 335, 126 Stevens, I. R., Forbes, D. A., \& Norris, R. P. 1999, MNRAS, 306, 479 Strickland, D. K., \& Stevens, I. R. 2000, MNRAS, 314, 511

Suchkov, A. A., Balsara, D. S., Heckman, T. M., \& Leitherer, C. 1994, ApJ, 430, 511

Telesco, C. M., \& Harper, D. A. 1980, ApJ, 235, 392

Thronson, H. A., Jr., \& Telesco, C. M. 1986, ApJ, 311, 98

Tomisaka, K., \& Ikeuchi, S. 1988, ApJ, 330, 695

Tschöke, D., Bomans, D. J., Hensler, G., \& Junkes, N. 2001, A\&A, in press

Tschöke, D., Hensler, G., \& Junkes, N. 1999, A\&A, 343, 373

Tschöke, D., Hensler, G., \& Junkes, N. 2000, A\&A, 360, 447

Tully, R. B. 1988, Nearby Galaxies Catalog (Cambridge: Cambridge University Press)

van der Kruit, P. C. 1973, A\&A 29, 231

Vogler, A., Pietsch, W., \& Kahabka, P. 1996, A\&A, 305, 74

Weaver, K. A., Heckman, T. M., \& Dahlem, M. 2000, ApJ, 534, 684

Wevers, B. M. H. R., van der Kruit, P. C., \& Allen, R. J. 1986, A\&AS, 66,505

Wynn-Williams, C. G., \& Becklin, E. E. 1985, ApJ, 290, 108

Zimmermann, H. U., Becker, W., Belloni, T., et al. 1997, EXSAS User's Guide, Edition 5, MPE Report, Garching 